\title{
MICROSPACECRAFT AND EARTH OBSERVATION: THE ELECTRICAL FIELD (ELF) MEASUREMENT PROJECT
}

\author{
F.J. Redd \\ T.A. Olsen \\ Utah State University \\ Logan, UT 84322
}

\begin{abstract}
Past attempts to map the earth's electrical field have been severely limited by the lack of simultaneous global measurements. Previous measurements have been made by sounding rocket and satellite borne sensors, but these measurements have covered only singular points in the field. These satellite observations are augmented by ground radar (incoherent scatter) plasma drift measurements; however, only six ground-based installations are producing such local electrical field maps. The expansion of this ground-based radar network to meet a global objective is politically and financially impossible. Global electrical field maps constructed by forcing mathematically formulated models to fit this limited set of data points are not only inaccurate, but the degree of inaccuracy is impossible to evaluate.
\end{abstract}

This paper discusses the design of a global electrical field sensing system to be deployed in a constellation of microspacecraft. Each microspacecraft incorporates a deployable sensor array ( $5 \mathrm{~m}$ booms) into a spinning oblate platform. Global deployment of $\mathbf{4 8}$ spacecraft is achieved through perturbation-driven dispersion of muliple spacecraft launched from eight Pegasus launch vehicles. The mass of each spacecraft is less than $25 \mathbf{~ k g}$, and the power requirements are less than $10 \mathrm{~W}$; all the required power can be generated by solar cells covering the exterior of the spacecraft. The program costs are estimated to be less than $\$ 100$ million.

\section{INTRODUCTION}

It is well known that the dynamic variations in the earth's electrical field have a strong influence on the physics of the upper atmosphere and can influence terrestrial atmospheric phenomena. Interactions between the electrical and magnetic fields and the solar wind often cause communication disruptions as well as observable phenomena such as the aurora. Efforts to understand and predict these influences and effects are 
hampered by the lack of accurate models of the earth's electrical field and its influence on the upper atmosphere. Past attempts to map this field have been severely limited by the lack of simultaneous global measurements. Sounding rocket and satellite borne sensors produce only singular measurements which are insufficient to verify current mathematical models. The models are therefore not only inaccurate, but the degree of inaccuracy is impossible to determine. The need for a globally deployed electrical field measurement system is clearly apparent. Although some data can be provided from ground based measurements, the expansion of the current ground radar system (6 installations) to meet global requirements is politically and financially impossible." The remainder of this paper will discuss a proposal for a space-based, global electrical field measurement system (ELF) which uses a constellation of microspacecraft as its primary element.

\section{MISSION DESCRIPTION}

The most dynamic electrical field activity around the earth occurs near the poles in the $50^{\circ}$ to $70^{\circ}$ latitude regions. It is in this region that phenomena such as the aurora are observed most frequently. Little activity of interest occurs in the equatorial regions. Therefore, the ELF satellites will be placed in orbits with inclinations between $45^{\circ}$ and $90^{\circ}$. This placement gives good coverage of the poles as well as some coverage of the equatorial regions.

The ELF satellites will be placed at altitudes between 500 and $1000 \mathrm{~km}$. In this range, the earth's electrical field does not change dramatically with altitude. Also, 500 $\mathrm{km}$ is high enough to give the lowest satellite a minimum mission life of 5 years before the orbit decays, and $1000 \mathrm{~km}$ is low enough to escape the radiation from the Van Allen belts.

Global constellation coverage begins with the launch of eight Pegasus vehicles, each containing six spacecraft. The initial eight orbits range between $55^{\circ}$ and $90^{\circ}$. Each cluster of six satellites will be separated into six individual orbits by capitalizing on the perturbation effects of the earth's oblateness.

The earth's oblateness causes an orbit's line of nodes to regress. The regression rate is a function of altitude and inclination. By slightly changing the relative orbit inclination or altitude of each satellite, slightly different orbital regression rates will be produced. Thus, the six satellite orbits will dispersed with time.

At lower orbital inclinations, a satellite has a larger moment arm for the equatorial mass to tug on. This causes the orbit to precess and the line of nodes to regress. The 
relationship which calculates the nodal regression per second for a circular orbit is given by the following equation. ${ }^{2}$ This is the averaged effect over one orbit.

$$
\dot{\Omega}--3 n J_{2} \frac{R^{2}}{2 a^{2}\left(1-e^{2}\right)^{2}} \cos i
$$

where

$$
\begin{array}{ll}
\mathrm{n} & =\left(\mu / \mathrm{a}^{3}\right)^{1 / 2} \\
\mu & =\text { earth gravitational } \\
& \text { parameter } \\
\mathrm{J}_{2} & =\text { Oblateness effect } \\
\mathrm{R} & =\text { Radius of earth } \\
\mathrm{i} & =\text { inclination } \\
\mathrm{a} & =\text { semi-major axis } \\
\mathrm{e} & =\text { eccentricity }
\end{array}
$$

A study of our orbit maneuvering capabilities shows that the maximum practical inclination difference between two satelites is about $0.5^{\circ}$. The maximum altitude difference between two circular orbiting satellites is about $75 \mathrm{~km}$. A comparison of regression rates achievable by the two methods is shown in Fig. 1. The top curve

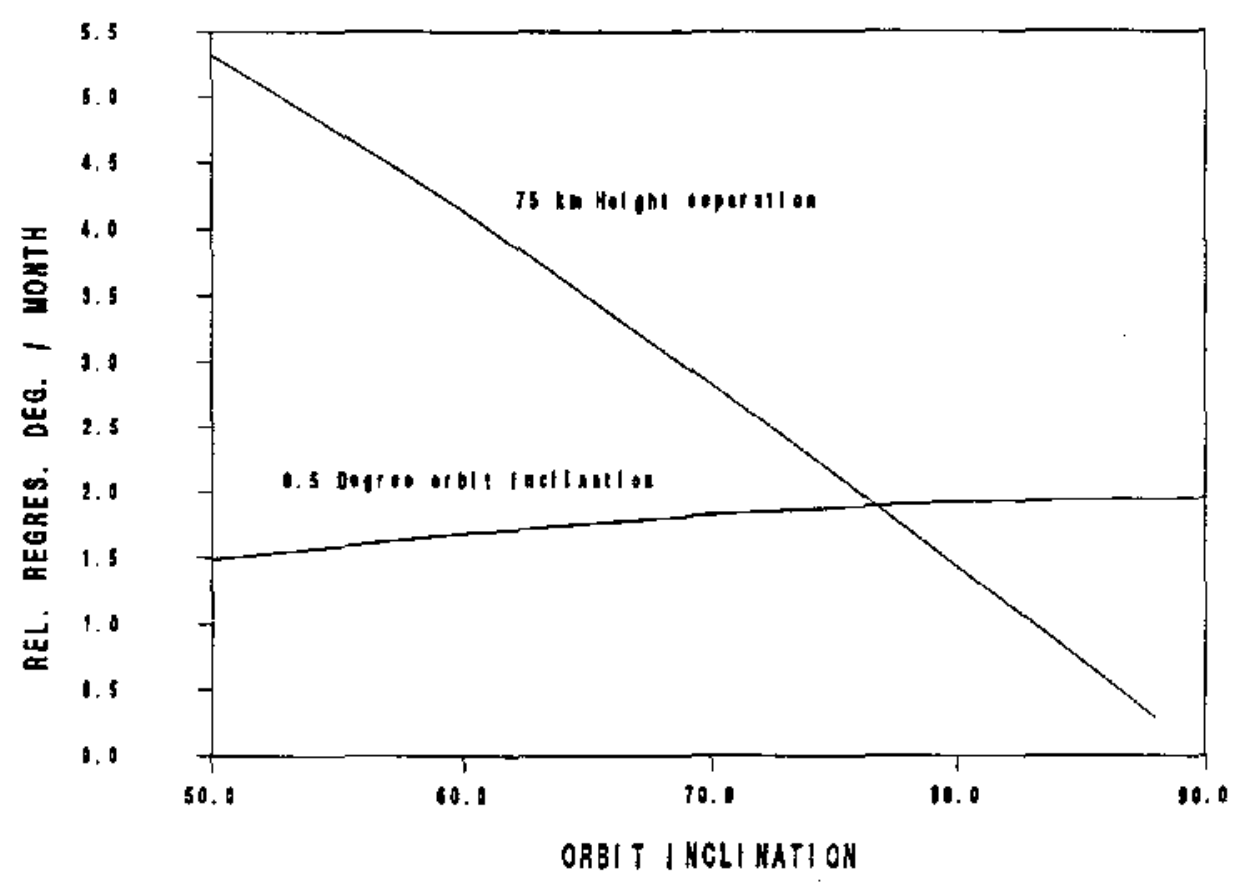

Fig. 1 Comparison of Altitude and Inclination Separation Methods 
shows regression rates for satellites separated by an altitude of $75 \mathrm{~km}$. The bottom curve shows regression rates for satellites with relative inclinations of $0.5^{\circ}$. It is evident from this graph that the altitude separation method is preferred from $75^{\circ}$ and below, and the inclination separation method is preferred from $75^{\circ}$ and above. In order to maximize the dispersion rate of the satellites, each of these two methods will be employed within its preferred range.

There are other considerations for each deployment method. For example, less fuel is required to perform the altitude maneuvers; the inclination dispersion requires about twice the fuel. Also, different altitudes cause each satellite to have a slightly different period. So, not only do the satellites' orbital planes gradually separate, but they quickly spread out along the orbit path. Complete dispersion around the earth takes place in about a day. For these reasons, the altitude separation method is more desirable; however, the inclination separation method is required near the polar regions.

When the initial orbit is reached, one of the six satellites will be ejected from the Pegasus upper stage. The rest of the cluster will remain attached. The stage will then be maneuvered to a slightly different altitude (about $75 \mathrm{~km}$ higher) or a slightly different inclination (about $2.5^{\circ}$ difference) where another ELF satellite will be ejected. This sequence will be repeated until all 6 satellites have been ejected. Launching the satellites in clusters like this reduces the required number of launches. Useful global dispersion will be achieved in about 4-6 months.

Upon insertion into orbit, a radio signal will be sent to the satelites to activate sensor boom deployment and spacecraft spin-up.

\section{ELECTRICAL FIELD SENSING PAYLOAD}

The electrical field sensing payload will consist of 3 orthogonal sets of insulated booms with conductive spheres attached to the ends. Three-dimensional electrical field data at a point will be obtained from measurement of the electric potential across each pair of conductive spheres. The boom system must be rotating in order to mitigate the photoelectric charge build-up caused by exposure to the sun. To achieve the desired accuracy, the spheres must be separated by at least 1 meter and be rotating at no more than 10 radians per second. Since the rotation causes a sinusoidal variation in the output, potential readings must be taken at least every 0.1 seconds to get the desired resolution.

The regions of interest to electrodynamicists lie above $50^{\circ}$ latitude at altitudes from 600 to $1000 \mathrm{~km}$. Typical electric field intensities in these areas are expected to range from $2 \mathrm{mV} / \mathrm{m}$ across the polar cap to $150 \mathrm{mV} / \mathrm{m}$ in the auroral regions. ${ }^{3}$ The ELF 
experiment will be capable of providing the global, three-dimensional data necessary to determine the nature of these fields.

The symmetric double probe technique has proven to be a reliable method of measuring ionospheric d.c. electric fields. ${ }^{4,5,6}$ The theory of such a system has been detailed by Fahleson. ${ }^{7}$

The operation of such a system is heavily dependant on the local electron temperature and density. ${ }^{8}$ High electron temperatures or low plasma densities may drive uncertainties in the data over acceptable limits. In regions of interest to us, average electron temperatures of $2100 \mathrm{~K}$ are expected with maximum temperatures of $8000 \mathrm{~K}$ during auroral events. Ion densities may change by several orders of magnitude.

The ELF satellite sensor configuration is shown in Fig. 2. The conducting portion of each boom consists of a gold plated aluminum sphere with a $5 \mathrm{~cm}$ radius. Four of

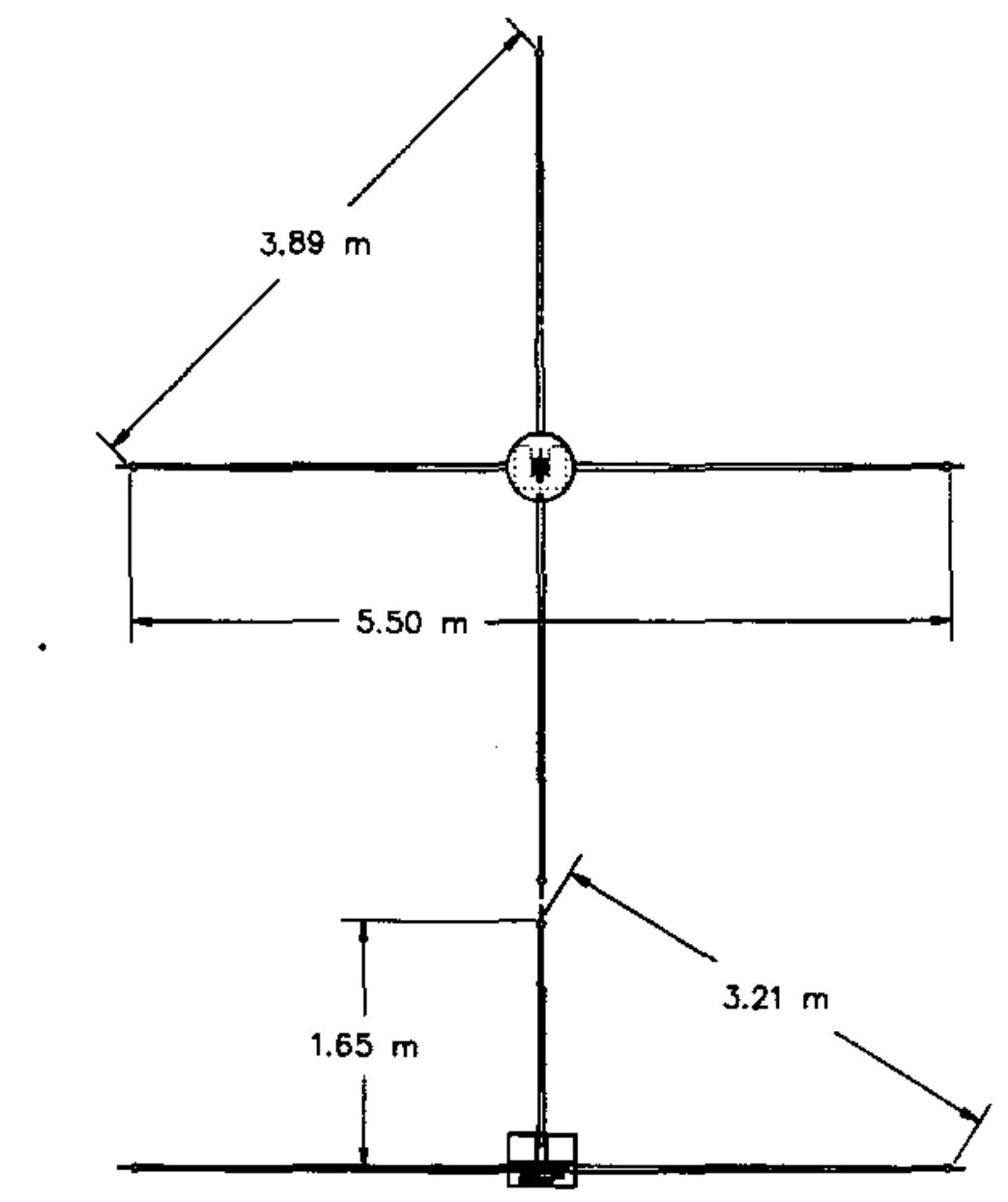

Fig. 2 ELF Sensor Configuration 
the spheres lie in the same plane and are supported by the $2.75 \mathrm{~m}$ Weitzmann Quadrupole Stacer boom system. ${ }^{9}$ The fifth sensor lies $1.65 \mathrm{~m}$ from the spacecraft body and is supported by a Weitzmann Monopole Stacer boom. All booms are composed of insulated beryllium copper. Stubs added to the end of the booms will minimize the photoelectric current and work function differences that could bias the system.

Probe voltages impressed across the high impedance $\left(1.2 \times 10^{9} \mathrm{ohm}\right)$ amplifiers (Burr-Brown AD515) are selected by the 8-1 analog multiplexer (Burr-Brown HI1-508A-2). The rate at which these signals are selected is variable and may be programmed to maximize data gathering efficiency. For instance, in some regions of particular interest the sample rate could be increased to maximize temporal resolution, and subsequently decreased to conserve memory. The 12 bit digital/analog converter (Burr-Brown ADC574) will produce a pre-programmed voltage, which, when compared with the signal from the multiplexer, will allow voltage biases to be removed. The dynamic range of this instrument is also variable and may be switched between $\pm 2.5 \mathrm{~V}, \pm 5 \mathrm{~V}$, and \pm 10 V. Thus, the 12 bit analog/digital converter (Burr-Brown ADC80AG) will allow an amplitudinal resolution of $0.6 \mathrm{mV} / \mathrm{m}, 1.2 \mathrm{mV} / \mathrm{m}$, or $2.4 \mathrm{mV} / \mathrm{m}$ from the $2.75 \mathrm{~m}$ booms, and a resolution of $1 \mathrm{mV} / \mathrm{m}, 2 \mathrm{mV} / \mathrm{m}$, or $4 \mathrm{mV} / \mathrm{m}$ from the $1.65 \mathrm{~m}$ boom. Total power required by this system is about 1 watt.

Data recorded by this sensor will be stored as common mode voltages from each probe. As opposed to differential voltages, data in this form will allow individual researchers some flexibility when resolving the electric field (e.g. each axis of the field may possibly be obtained from any arbitrary combination of probe voltages). This ability will also provide redundancy in the information from which the ambient electric field can be obtained.

Spinning the spacecraft allows easy evaluation of any voltage biases inherent in the system, ${ }^{5}$ and provides some information about the probe location with respect to the spacecraft. A rotating spacecraft also eliminates the possibility of one boom remaining in the spacecraft wake or shadow for an extended period. For these reasons, it was required that the ELF satellites be given an angular velocity of $1.05 \mathrm{rad} / \mathrm{s}$ about the 1.65 $m$ boom (the maximum axis of inertia).

Errors in the electrical field measurements taken by the ELF satellites may arise from many effects; contact potential differences caused by variations in the work function associated with each probe can cause voltage errors as large as several hundred millivolts. ${ }^{5}$ Care must be taken in the construction and deployment of the probes to minimize the geometric differences and contamination effects that lead to this problem. Contact potentials and other biasing effects, if relatively constant throughout the orbit. 
may be evaluated using measurements taken in regions where $\mathbf{E}$ is expected to be near zero.

Errors in attitude evaluation will cause a corresponding error in the determination of the $\mathbf{V} \times$ B electrical field component in satellite's frame of reference. This component will be evaluated using data collected by the magnetometer and other attitude sensing instruments. Every degree of uncertainty in attitude determination or knowledge of boom orientation can cause an uncertainty of up to $1 \%$ in $\underline{\mathbf{V}} \times \mathbf{B}$.

Another source of error in the potentials recorded by the ELF satellites is the inhomogeneities in the temperature and density of the plasma through which the sensors are moving. Differences in probe velocities caused by the rotation of the satellite will contribute to this error.

The booms supporting the conducting probes can cause some differences in plasma density and temperature by screening particles from the conducting portion of the probe. Thermal noise from the input amplifiers will cause additional uncertainty in the measurement.

The total error in the electric field measurement will be the sum of the above errors and any errors arising from other effects. This error is expected to be about $10 \%$ $-20 \%$, depending on the accuracy with which individual errors can be interpreted.

Photoelectric current differences caused by unequal shading or plasma inhomogeneities caused by the spacecraft wake or high energy particle fluxes may occasionally upset potential readings. However, due to the form in which these potential readings are stored (ie. common mode voltages), data points recognized as having been recorded during one of these events may often be discarded with no loss of continuity in the electric field constructed for that orbit.

\section{ATTITUDE CONTROL AND DETERMINATION SYSTEM}

The satellite is modelled as a spinning oblate platform which spins at $10 \mathrm{rpm}$. The orientation or attitude of each satellite will not be controlled, but the spin rate will be controlled to $10 \pm 2 \mathrm{rpm}$. An on-board cold gas propellant system will be used to spinup the satellite initially as well as make any necessary spin rate adjustments.

Two 2-axis magnetometers made by lthaco, 2 Lockheed Wide Angle Sun Sensors (WASS), and 1 thaco Bullseye Horizon Crossing Computer (BHCC) are used to determine the attitude of each ELF satellite. By using different combinations of these 5 sensors, the attitude of the satelite can be determined at all times during the orbit. 
Some of the data will be redundant, but this redundancy can be used to enhance the accuracy of the readings. Although a complete error analysis has not yet been performed, it is believed that attitude readings will lie within the $\pm 1^{\circ}$ error in attitude knowledge margin.

The position of the sensors was a critical factor in the design. The BHCC is placed $90^{\circ}$ off the spin axis in order to insure horizon scanning. This position will work in all orientations except when the spin axis is exactly parallel with the horizon. This can occur only briefly during each orbit. This will not affect the overall attitude determination because the sun sensors and the magnetometers can give the attitude, albeit to a lesser degree of accuracy. The two sun sensors are placed on the top and bottom of the spacecratt in order to ensure that one of the sensors can find the sun as the horizon is being scanned by the BHCC. The two 2-axis magnetometers will be placed on the opposite side of the electronics to reduce any false readings due to magnetic fields.

\section{DATA PROCESSING SYSTEM}

The data processing system provides control and processing for the active satellite subsystems. These include electrical field sensing, attitude sensing and spin-rate control, communications, and power control. In addition, the data processing system will perform various housekeeping tasks such as monitoring internal temperatures and NiCad cell states of charge.

The ELF satellite acquires electrical field samples periodically, at known intervals and at known attitudes. Samples, in the form of voltages, are converted to a digital representation. Along with timing and attitude information, these samples are stored on board the satellite, to be forwarded to earth twice daily. Other "state-of-the-satellite" information is appended to the transmission as requested. The data processing system supports these functions.

In addition, the satellite operation may need to be altered on command from earth. The system provides that flexibility. For example, the electrical field samples can be offset and scaled as desired, the operating software can be modified, and NiCad cell banks can be disconnected from the main power bus for cycling to restore capacity.

A basic data processing or microprocessor system will include a microprocessor, external memory, and simple logic functions (these functions direct, gate, store, and invert signals to assure correct system operation and are often known as "glue logic"). Often, where large amounts of data must be stored, a mass storage device or system is included. Peripheral components, which reduce the need for microprocessor attention by independently performing functions such as input/output $(I / O)$ and timing, may also be used. An integrated approach to this microprocessor system encompasses the other satellite systems to a degree, because of the interdependence of the systems. 
The ELF microprocessor system comprises a processor (Harris $80 \mathrm{C} 86$ ), general purpose processor memory (64k PROM, 128k EEPROM, 256k SRAM), data storage memory (8M SRAM), and peripherals including a communications interface (NSC DP8390C), a programmable timer (Harris 82C54), a watchdog timer, and a programmable interrupt controller (Harris $82 \mathrm{C59}$ ).

The data processing system is sized to store up to 24 hours worth of data. These data include the electrical field potential as well as the attitude readings.

\section{COMMUNICATION SYSTEM}

Because the attitude of each ELF satellite will not be controlled and each satellite can maintain a different orientation, a virtually omni-directional spacecraft antenna is needed for up-link and down-link communications. The Physical Science Laboratory stripline wraparound antenna Model 55-XXX meets this requirement. A frequency of $2200 \mathrm{MHz}$ provides sufficient bandwidth for both for receiving instructions and transmitting the collected data to a ground station. The ELF satellite uses the AydinVictor Model T-1024S transmitter. Data will be transmitted twice per day to one ground station.

The ground station will use the $4.3 \mathrm{~m}$ parabolic dish with tracking capabilities (Model 2P14) manufactured by Anderson Manufacturing. The actual location of this ground station is yet to be determined. Each satellite will take a maximum of 82 seconds to transmit the stored data. It is expected that each satellite will pass within range of the ground station at least twice per day.

The transfer of information from one point in space to another using electromagnetic wave propagation requires a transfer of energy. Each operation in the data link contributes a gain or a loss to the energy transfer. These operations can be expressed as an equation that will detail the data link budget. ${ }^{10}$

In quantifying the link budget, we use an equation that yields a margin of safety or signal margin $(M)$ :

$$
M=\frac{P_{t} G_{t} G_{r}}{\left(E_{b} / N_{o}\right) k T B L_{\sigma} L_{\sigma} L_{0}}
$$

where

$$
\begin{aligned}
P_{\mathrm{t}} & =\text { power of transmitter } \\
\mathrm{G}_{\mathrm{t}} & =\text { gain of transmitter } \\
\mathrm{G}_{\mathrm{r}} & =\text { gain of receiver } \\
\left(\mathrm{E}_{\mathrm{b}} / N_{\mathrm{o}}\right)_{\mathrm{req}} & =\text { required bit error rate } \\
\mathrm{B} & =\text { bandwidth } \\
\mathrm{k} & =\text { Boltzmann's constant }\left(1.38 \times 10^{-23}\right)
\end{aligned}
$$




$$
\begin{aligned}
& \mathrm{T} \\
& \mathrm{L}_{\mathrm{s}} \quad=\text { receiving noise temperature (degrees kelvin) } \\
& \mathrm{L}_{\mathrm{a}} \quad=\text { atmospheric attenuation of the wave } \\
& \mathrm{L}_{0} \quad=\text { non-ideal system components loss }
\end{aligned}
$$

Using the transmitter and receiver stated above and frequency shift keying, the least efficient digital signal modulation method, the signal margin for the ELF data link is $5 \mathrm{~dB}$ at $2200 \mathrm{MHz}$. This is the worst case. There are other modulation/demodulation schemes that would yield a higher signal margin.

\section{POWER SYSTEM}

The each satellite's power will be supplied by silicon dioxide solar cells wrapped around the exterior of the spacecraft. Since the satellites will not generally be placed in sun synchronous orbits, they will have to function in the dark as well. Therefore, the solar cells will be backed up with 60 AA-sized NiCad batteries connected in series to supply $12 \mathrm{~V}$. The minimum end-of-life power generation will be $12.77 \mathrm{~W}$ which will be sufficient to cover the power requirements of all systems.

\section{ELF SATELLITE STRUCTURE AND CONFIGURATION}

The cylindrical primary structure is $45 \mathrm{~cm}$ in diameter and $35 \mathrm{~cm}$ high. It will be composed of 0.16 to $0.32 \mathrm{~cm}$ thick aluminum 6061-T6. The primary support plate will be constructed of $1.25 \mathrm{~cm}$ thick aluminum honeycomb. The subsystem components will be mounted on this plate as shown by Fig. 3. Individual component covers will provide radiation shielding as required.

Several considerations were made in the placement of the subsystems. Some of the subsystems require a specific location, others allow some variance in location. For the latter, dimensional constraints and the location of center of mass dictated their position.

The sensors which are included within the attitude sensing and control system require definite locations. Two sun sensors, each located on a flat side of the satellite, have a mass of 132 grams and dimensions of $6.6 \times 5.8 \times 5.1 \mathrm{~cm}$ each. One horizon sensor, located on the perimeter of the satellite, has a mass of $300 \mathrm{grams}$ and dimensions of $12.7 \times 5.8 \times 3.8 \mathrm{~cm}$. Two magnetometers, placed against the outer skin of the satellite to prevent interference from circuit electronics, have a mass of 350 grams and dimensions of $4.3 \times 13.3 \times 6.7 \mathrm{~cm}$.

The circuit board is mounted on the honeycomb plate to take advantage of the aluminum in the plate to shield against radiation. A radiation shield of milled aluminum 
which weighs $1.35 \mathrm{~kg}$ gives the board a $0.5 \mathrm{~cm}$ thick protective shield to the remaining sides.
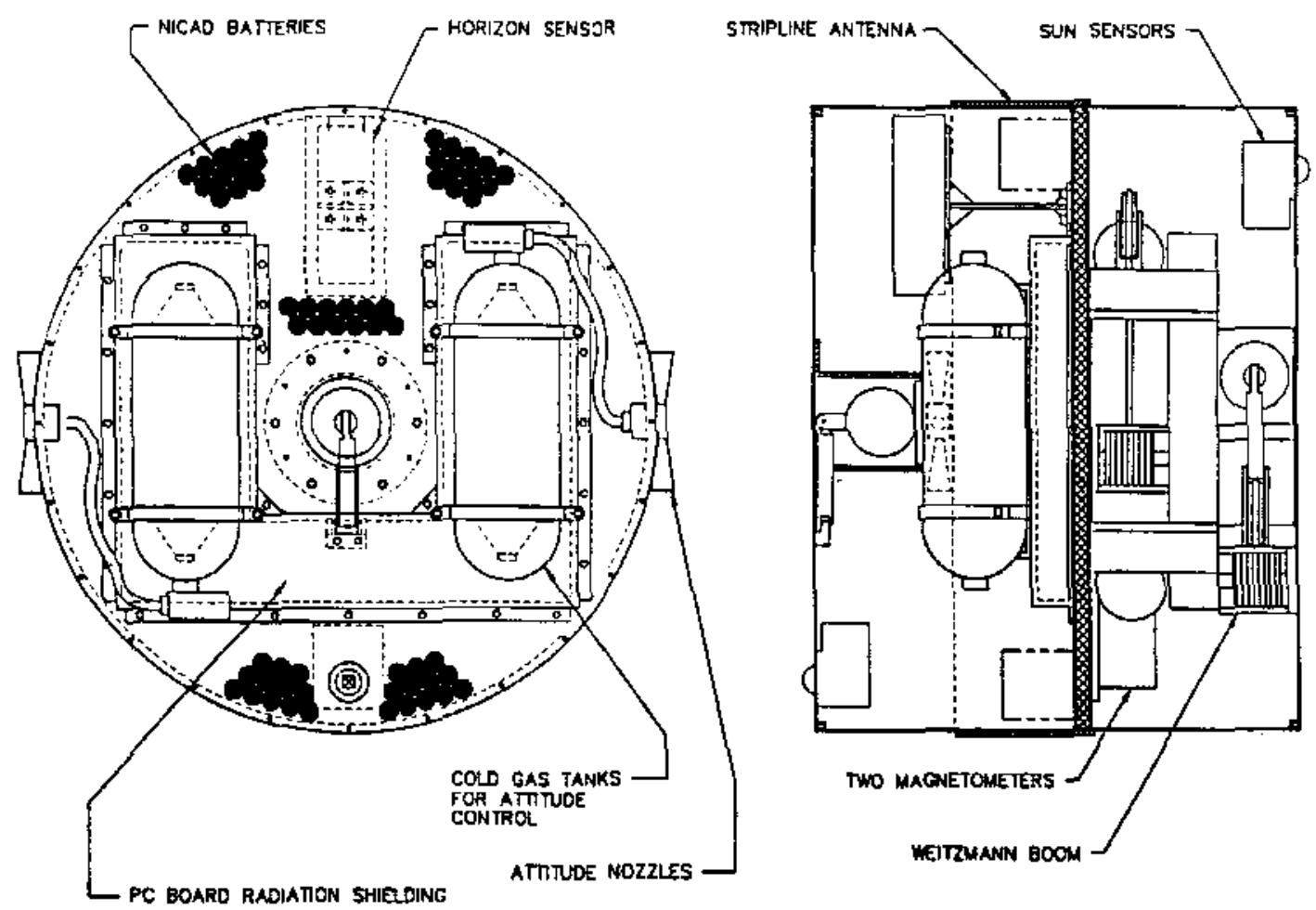

Fig. 3 Internal Layout of the Subsystem Components

Two cold gas tanks are mounted to the circuit board radiation shield. Each tank has a dry mass of $0.25 \mathrm{~kg}$ and will initially contain $2 \mathrm{~kg}$ of $\mathrm{N}_{2}$. The tanks have a length of $23 \mathrm{~cm}$ and a diameter of $7.5 \mathrm{~cm}$ each.

The batteries for the power subsystem consist of 5 groups of 12 AA-size batteries. The flexibility in placement of the battery packs allows us to use them as balance weights. Situating the batteries as shown in figure 9.5 , we are able to locate the center of mass along the spin axis.

The stripline antenna is made of a $2 \mathrm{~mm}$ thick by $100 \mathrm{~mm}$ wide copper strap. It is located on the perimeter of the satelite and has a mass of $0.1 \mathrm{~kg}$.

\section{THERMAL CONTROL SYSTEM}

In order to maintain a desirable temperature for all components, the temperatures of each ELF satellite must stay within $270 \mathrm{~K}$ to $320 \mathrm{~K}$. We considered two steady state extreme cases for initial modelling. The first, the hottest orbit, is one in which one flat 
which weighs $1.35 \mathrm{~kg}$ gives the board a $0.5 \mathrm{~cm}$ thick protective shield to the remaining sides.
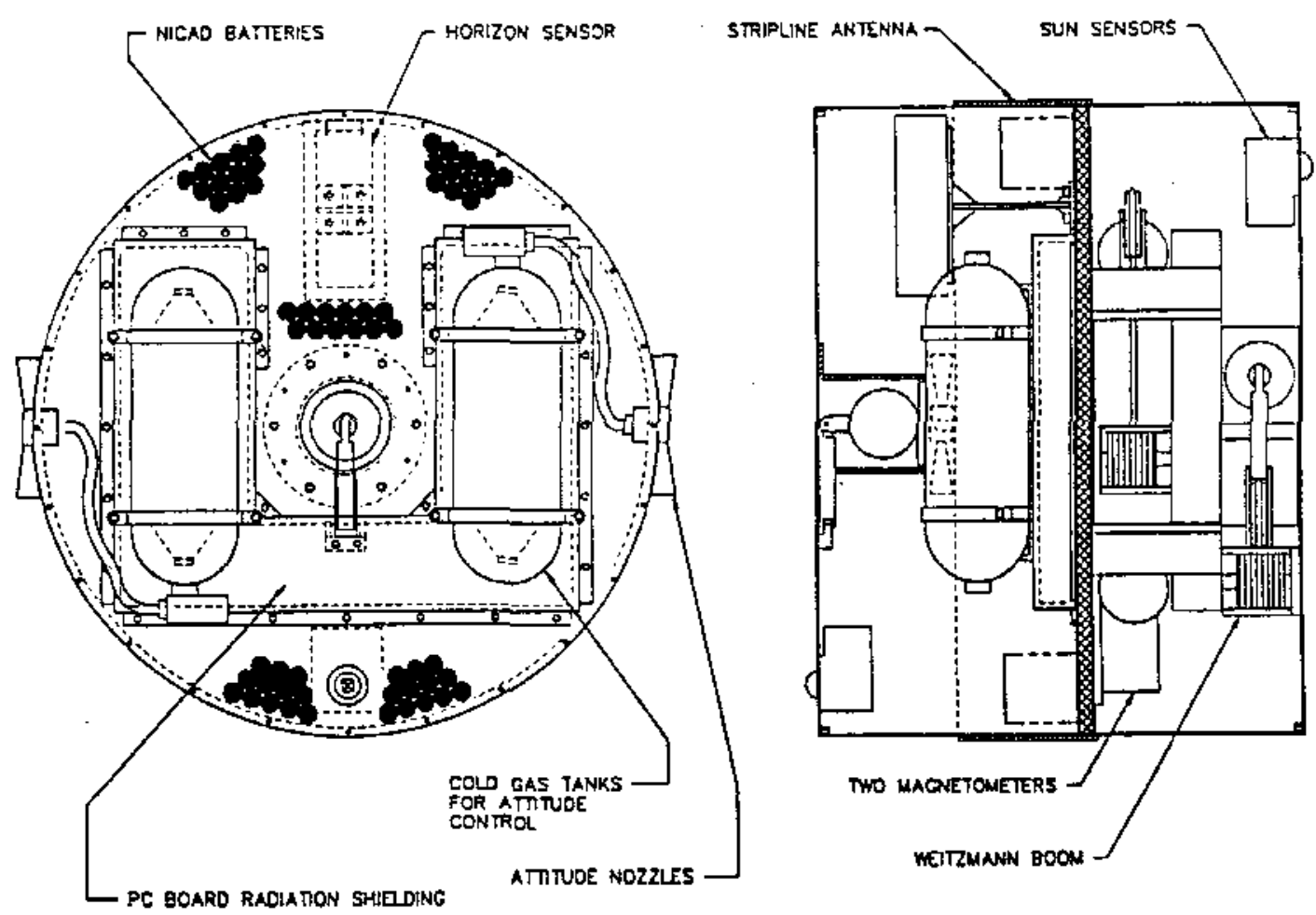

Fig. 3 Internal Layout of the Subsystem Components

Two cold gas tanks are mounted to the circuit board radiation shield. Each tank has a dry mass of $0.25 \mathrm{~kg}$ and will initially contain $2 \mathrm{~kg}$ of $\mathrm{N}_{2}$. The tanks have a length of $23 \mathrm{~cm}$ and a diameter of $7.5 \mathrm{~cm}$ each.

The batteries for the power subsystem consist of 5 groups of 12 AA-size batteries. The flexibility in placement of the battery packs allows us to use them as balance weights. Situating the batteries as shown in figure 9.5, we are able to locate the center of mass along the spin axis.

The stripline antenna is made of a $2 \mathrm{~mm}$ thick by $100 \mathrm{~mm}$ wide copper strap. It is located on the perimeter of the satellite and has a mass of $0.1 \mathrm{~kg}$.

\section{THERMAL CONTROL SYSTEM}

In order to maintain a desirable temperature for all components, the temperatures of each ELF satellite must stay within $270 \mathrm{~K}$ to $320 \mathrm{~K}$. We considered two steady state extreme cases for initial modelling. The first, the hottest orbit, is one in which one flat 
face of the satellite constantly faces the sun. If an ELF satellite winds up with this orientation, all on-board components will stay within their temperature ranges. The second case examined a coldest orbit in which the flat faces of the satellite never see the sun, and the spacecraft passes through the earth's shadow. In this orbit some component temperatures descended below their specified limits. To compensate for this, three $1 \mathrm{~W}$ heaters will be used to bring the components into their required operating temperature range. These heaters are equipped with their own temperature regulating switches.

\section{CONCLUSIONS}

Preliminary estimates indicate that the mass of each satellite will be $19.1 \mathrm{~kg}$. Hardware cost for each will be less than $\$ 250,000$. Including deployment costs, the program will cost about $\$ 70$ million for 8 Pegasus launches and 48 satellites. Labor costs have not yet been computed.

The proposed ELF system envisions the deployment of about 50 satellites. The number of spacecraft in the constellation provides an inherent redundancy in the system. Even the loss of a launch vehicle with its load of six satellites would not cause a drastic degradation of the system. The system of microsatellites is also resilient to budget fluctuations. We expect to lose not more than $10 \%$ of the satellites to launch vehicle malfunction, ELF subsystem failure, or space debris impact.

The ELF electrical field measurement system with its space and ground elements is seen as an effective means of providing accurate measurements of the earth's electrical field. It's relatively low cost and its robustness suggest that it should be seriously considered as a potential element in the Mission to Planet Earth initiative.

\section{ACKNOWLEDGEMENTS}

The authors wish to thank Dr. Jan J. Sojka for his contributions to the research effort that generated this paper.

The ELF design project was conducted through funding under the NASA/USRA Advanced Design Program. 


\section{REFERENCES}

1. Sojka, J.J., "2-DEF: A Two Dimensional Electric Field Mission", CASS Report No. 89-5-2, Center for Atmospheric and Space Sciences, Utah State University, Logan, UT, November 1989.

2. Wiesel, W.E., Spaceflight Dynamics (eq. 3.53), McGraw-Hill, 1989.

3. Sojka, J.J., private communication, 1990.

4. Mozer, F.S., "Analyses of Techniques for Measuring D.C. and A.C. Electric Fields in the Magnetosphere", Space Sci Rev, 14:272-313, 1973.

5. Fahleson, U.V., Kelly, M.C., and Mozer, F.S., "Investigation of the Operation of a D.C. Electric Field Detector", Planet. Space Sci, 22:777-789, 1978.

6. Heppner, J.P., Maynard, N.C., and Aggson, T.L., "Early Results from ISEE-1 Electric Field Measurements", Space Sci Rev, 22:777-789, 1978.

7. Fahleson, U.V., "Theory of Electric Field Measurements Conducted in the Magn etosphere with Electric Probes", Space Sci Rev, 7:238-262, 1967.

8. Cauffman, D.P. and Gurnett, D.A., "High Latitude Convection Electric Fields", Space Sci Rev, 13:369-410, 1972.

9. Weitzmann, R.H., Correspondence, Weitzmann Consulting, Inc., Aerospace Engineering, San Francisco, CA, April, 1990.

10. Sperry, Data Link Basics - The Link Budget, p.1. 